\title{
Decay characteristics of two-dimensional islands on strongly anisotropic surfaces
}

\author{
Yugui Yao, ${ }^{1}$ Ph. Ebert, ${ }^{2}$ Maozhi Li, ${ }^{1}$ Zhenyu Zhang,,${ }^{3,1}$ and E. G. Wang ${ }^{1}$ \\ ${ }^{1}$ International Center for Quantum Structures and Institute of Physics, Chinese Academy of Sciences, \\ Beijing 100080, People's Republic of China \\ ${ }^{2}$ Institut für Festköperforschung, Forschungszentrum Jülich GmbH, 52425 Jülich, Germany \\ ${ }^{3}$ Solid State Division, Oak Ridge National Laboratory, Oak Ridge, Tennessee 37831-6032 \\ (Received 19 February 2002; revised manuscript received 17 May 2002; published 30 July 2002)
}

\begin{abstract}
We investigate analytically the decay characteristics of two-dimensional islands on strongly anisotropic surfaces. We show that a generic scaling law can always be established in describing the dynamical evolution of such islands, given by $L \propto\left(t_{0}-t\right)^{\beta}$, where $L$ is the island width, and $t_{0}$ is the lifetime of the islands. The value of the scaling exponent $\beta$ in the fast-decay direction is always $1 / 2$ in the low-temperature regime where the decay is quasi-one-directional, irrespective of the specific dominant decaying mechanism involved. At higher temperatures where the decay proceeds effectively in both directions, $\beta$ is again a good measure of the dominant microscopic decaying mechanism involved, just like the isotropic case. We discuss these results in connection with recent experiments.
\end{abstract}

DOI: 10.1103/PhysRevB.66.041407

PACS number(s): 68.55.-a, 68.60.Dv

There has been an overwhelming wealth of research activity on fabricating various nanostructures that may possess unique physical properties. Nevertheless, the stability of nanostructures, once formed, often poses a limiting factor for their practical applications. ${ }^{1,2}$ Therefore, understanding the physical mechanisms involved in the stability and the dynamical evolution of nanostructures after their formation is both scientifically intriguing and technologically significant, and has been receiving increasing attention in recent years. One of the widely used model systems for such investigations is the decay of two-dimensional (2D) metal islands on metal substrates. ${ }^{2-11}$ The thermal decay of such islands was found to reduce the island area with time $t$ proportional to $\left(t_{0}-t\right)^{2 \beta}$, where $t_{0}$ is the time at which the island is fully dissolved. ${ }^{12}$ The value of the exponent $\beta$ is a signature of the dominant microscopic mechanism governing the rate of decay. In the case where attachment and detachment of atoms from the island edges dominate the rate of decay, $\beta$ equals $1 / 2$ whereas for the diffusion-limited case, i.e., where diffusion of adatoms on the terrace limits the decay rate, $\beta$ is $1 / 3$. These results very well describe the decay of islands on isotropic surfaces, which in fact has attracted most of the attention so far. However, the decay of islands on anisotropic substrates, i.e., surfaces with different energy barriers along the nonequivalent crystallographic directions, is expected to be more complex and may provide additional insight into the physical nature of island dissolution. Indeed the larger complexity was substantiated by the observation of a transition from a 2D decay mode at high temperatures to a quasi-onedimensional (1D) decay mode at low temperatures for the anisotropic system of Ag islands on $\mathrm{Ag}(110) .{ }^{11}$ Several more recent studies have also studied the evolution of islands and voids on other metal (110) surfaces, ${ }^{13-15}$ but to date, the theoretical basis for description of island decay on anisotropic substrates is still lacking.

In this paper we develop a comprehensive analytical description of the decay of two dimensional islands on highly anisotropic surfaces. We find that even for highly anisotropic surfaces the scaling law describing the decay of the islands' length $l \propto\left(t-t_{0}\right)^{\beta}$ is valid, but the exponent $\beta$ is significantly different as compared to isotropic surfaces: At low temperatures the model yields a quasi-one-dimensional island decay, i.e., $\beta$ is 0 along the slow-decay direction and $1 / 2$ along the fast-decay direction for both the attachment-detachment and the diffusion-limited regimes, in contrast to island decay on isotropic surfaces. At high temperatures, the islands decay two dimensionally and $\beta$ is $1 / 2$ and $1 / 3$ in the attachmentdetachment and diffusion-limited regimes, respectively, similar to the island dissolution on isotropic surfaces. Application of the present model to recent experimental data yields improved agreement.

Let us consider a rectangular monatomic-layer-high island on an anisotropic surface surrounded by the adatom vapor as shown in Fig. 1, where $2 L_{x}$ and $2 L_{y}$ are the length and width of the island edges in $x$ and $y$ directions, respectively. The two nonequivalent crystallographic directions of the substrate, $x$ and $y$, have different migration energy barriers. In

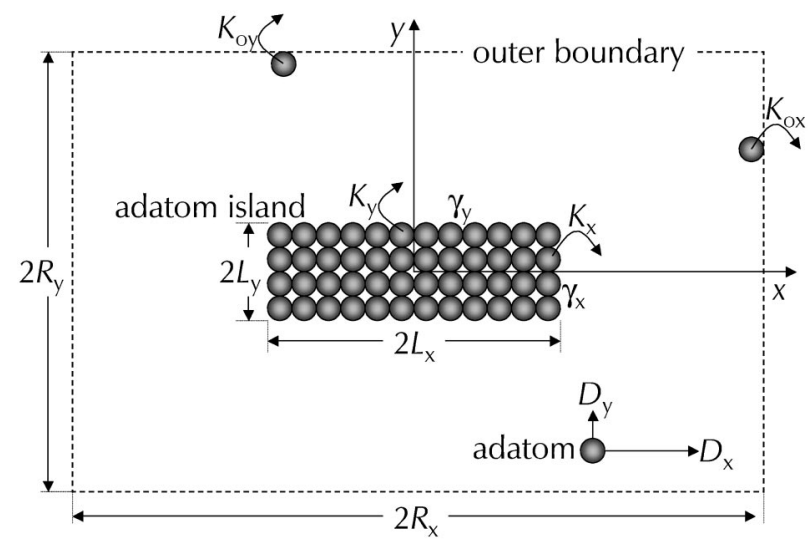

FIG. 1. Schematic of the different processes occurring during the decay of an adatom island on an anisotropic surface. The subscripts $x$ and $y$ denote two different crystallographic directions. $\gamma_{x}$ and $\gamma_{y}, K_{x}$ and $K_{y}, K_{o x}$ and $K_{o y}$, and $D_{x}$ and $D_{y}$ are the step line tensions, the kinetic coefficients at island step, the kinetic coefficients at outer boundary, and the diffusion coefficients, respectively. 
case of the (110) surfaces of fcc metals this leads to an "easy" in-channel diffusion along the $\langle 110\rangle(x)$ direction and a "difficult" cross channel diffusion in the orthogonal $\langle 100\rangle$ (y) direction. ${ }^{16}$ The decay of such an island is inherently coupled to a mass flow from the island to the surrounding environment. Three successive atomic processes are involved: (i) the interface transfer of atoms at the island edge, i.e., detachment and attachment events, (ii) the adatom diffusion on the surface, and (iii) the incorporation of the adatoms into the environment at the outer boundary, i.e., at sinks. $^{12}$

A decaying island must have a net detachment of atoms from the island. Thus the atom current $I_{i}$ is $I_{i}\left(L_{x}, y\right)$ $=K_{x}\left[\rho_{L_{x}}^{e q}-\rho\left(L_{x}, y\right)\right]$ and $I_{i}\left(x, L_{y}\right)=K_{y}\left[\rho_{L_{y}}^{e q}-\rho\left(x, L_{y}\right)\right]$ in the $x$ and $y$ directions at island edges, respectively. $K_{x}$ and $K_{y}$ are the rate coefficients for the attachment-detachment events at the island edges, which are assumed to be independent of the island size. $\rho(x, y)$ is the density of the adatom vapor on the surface and $\rho_{L_{x}}^{e q}$ and $\rho_{L_{y}}^{e q}$, referring to Wulff's construction, ${ }^{17}$ are the adatom densities at which the adatom vapor is in equilibrium with the condensed phase. They are given by $\rho_{L_{x}}^{e q}=\rho_{x, \infty}^{e q} \exp \left[2 \gamma_{x} \Omega /\left(L_{x} k T\right)\right]$ and $\rho_{L_{y}}^{e q}=\rho_{y, \infty}^{e q} \exp \left[2 \gamma_{y} \Omega /\left(L_{y} k T\right)\right]$, with $\rho_{x, \infty}^{e q}$ and $\rho_{y, \infty}^{e q}$ being the equilibrium vapor densities, $\gamma_{x}$ and $\gamma_{y}$ the free energies per unit step length (also called line tension), and $\Omega$ the area occupied by one atom in the condensed phase.

Once detached from a decaying island, an adatom migrates on the terrace. In a quasi-steady state approximation, the adatom concentration $\rho(x, y)$ satisfies

$$
D_{x} \frac{\partial^{2} \rho(x, y)}{\partial x^{2}}+D_{y} \frac{\partial^{2} \rho(x, y)}{\partial y^{2}}=0
$$

with $D_{x}$ and $D_{y}$ being the diffusion coefficients in the $x$ and $y$ directions, respectively.

Finally, the adatoms must also leave the vapor by crossing the outer boundary, i.e., by being incorporated into the environment. Similar to the case of interface transfer, the atom currents across the outer boundary are $I_{b}\left(R_{x}, y\right)$ $=K_{o x}\left[\rho\left(R_{x}, y\right)-\rho_{R_{x}}^{e q}\right]$ and $I_{b}\left(x, R_{y}\right)=K_{o y}\left[\rho\left(x, R_{y}\right)-\rho_{R_{y}}^{e q}\right]$ for the $x$ and $y$ directions, respectively. These equations introduce the rate coefficients $K_{o x}$ and $K_{o y}$ as well as the lengths $R_{x}$ and $R_{y}$ of the outer boundary.

In a steady-state system, the atom currents arising from the interface transfer at the island edges, the diffusion on the terrace, and the incorporation at the outer boundary must be equal. This provides the boundary condition to Eq. (1).

Up to now all the derivations are general and rigorous. In the following we focus on the case of island decay on a strongly anisotropic surface. Introducing the transformations $\Gamma=\left(D_{y} / D_{x}\right)^{1 / 2}, \quad u=\Gamma x$, and $v=y$ yields for Eq. (1) $\nabla^{2} \rho(u, v)=0$, which is a standard Laplace's equation. The different parameters in the new $(u, v)$ space are $L_{u}=\Gamma L_{x}$, $L_{v}=L_{y}, \quad R_{u}=\Gamma R_{x}, R_{v}=R_{y}, \quad D_{u}=\Gamma^{2} D_{x}, \quad D_{v}=D_{y}, K_{u}$ $=\Gamma K_{x}, K_{v}=K_{y}, K_{o u}=\Gamma K_{o x}, K_{o v}=K_{o y}, \gamma_{u}=\Gamma \gamma_{x}$, and $\gamma_{v}=\gamma_{y}$. If $D_{x}$ is sufficiently larger than $D_{y}$ such that $L_{v}$ $\gg L_{u}$ (this condition is fullfilled in the experiment, see Refs. 11 and 14), the shape of the still rectangular island in the $(u, v)$ space is close to an ellipse whose major and minor axes are $2 L_{v}$ and $2 L_{u}$, respectively. To the first order of approximation, the corresponding solution of the Laplace's equation, by adopting ellipse boundary conditions, is

$\rho(u, v)=A \times \operatorname{arccosh}\left(\frac{\sqrt{u^{2}+(v-c)^{2}}+\sqrt{u^{2}+(v+c)^{2}}}{2 c}\right)+B$,

where $c=\left(L_{v}^{2}-L_{u}^{2}\right)^{1 / 2}$. $A$ and $B$ are constants, which can be determined by setting the three kinds of total mass flow currents to be equal.

Back transformation of Eq. (2) yields the adatom density $\rho(x, y)$ in the $(x, y)$ space, from which the time variation of the island widths $L_{x}$ and $L_{y}$ can be obtained:

$$
\begin{gathered}
L_{y} \frac{d L_{x}}{d t}=-\Re \arctan \left[\left(\frac{\Gamma L_{x}}{L_{y}}\right)^{-1 / 2}\right], \\
L_{x} \frac{d L_{y}}{d t}=-\Re \arctan \left[\left(\frac{\Gamma L_{x}}{L_{y}}\right)^{1 / 2}\right]
\end{gathered}
$$

with

$$
\mathfrak{R}=\Omega \frac{\alpha_{R}^{-1}\left(\rho_{L_{y}}^{e q}-\rho_{R_{y}}^{e q}\right)+\left(\rho_{L_{y}}^{e q}-\rho_{R_{x}}^{e q}\right)+\frac{\alpha}{\alpha_{R}}\left(\rho_{L_{x}}^{e q}-\rho_{R_{y}}^{e q}\right)+\alpha\left(\rho_{L_{x}}^{e q}-\rho_{R_{x}}^{e q}\right)}{\frac{£}{\sqrt{D_{x} D_{y}}}+(1+\alpha) \frac{1}{K_{o x} R_{o y}}+\left(1+\alpha_{R}^{-1}\right) \frac{1}{K_{y} L_{x}}} .
$$

The leading term of $£$ is $(\alpha+1)\left(\alpha_{R}^{-1}+1\right)\left(\operatorname{arccosh}\left(b_{R} / c\right)\right.$ $\left.-\operatorname{arccosh}\left\{\left[L_{v} /\left(L_{v}-L_{u}\right)\right]^{1 / 2}\right\}\right)$, with $2 b_{R}=\left[R_{u}^{2}+\left(R_{v}-c\right)^{2}\right]^{1 / 2}$ $+\left[R_{u}^{2}+\left(R_{v}+c\right)^{2}\right]^{1 / 2}, \quad \alpha=\left(K_{x} L_{y}\right) /\left(K_{y} L_{x}\right), \quad$ and $\quad \alpha_{R}$ $=\left(K_{o x} R_{y}\right) /\left(K_{o y} R_{x}\right)$.

In order to get a more explicit dependence of $L_{x}$ and $L_{y}$ on the time $t$, two further approximations are adopted: (i) the equilibrium densities $\rho_{R x}^{e q}$ and $\rho_{R y}^{e q}$ at the outer boundary are close to the equilibrium adatom densities $\rho_{x, \infty}^{e q}$ and $\rho_{y, \infty}^{e q}$, respectively. This is valid for low island densities, i.e., $R_{x}, R_{y} \gg L_{x}, L_{y}$. (ii) $\rho_{L_{x}}^{e q}$ and $\rho_{L_{y}}^{e q}$ are replaced by the constant and linear terms of their Taylor expansions. Here, we focus on the cases of strongly anisotropic surfaces, where 
$D_{x} \gg D_{y}$. Considering that the attachment energy barriers are generally similar to diffusion barriers, we should have $K_{x} \gg K_{y}$ and $K_{o x} \gg K_{o y}$ if $D_{x} \gg D_{y}$. This leads to $\alpha \gg 1, \alpha_{R}$ $\gg 1$, and $\left(D_{x} / D_{y}\right)^{1 / 4}\left(L_{y} / L_{x}\right)^{1 / 2} \gg 1$. Using these simplifications, the scaling laws for the decay of islands can be established for different physical conditions.

Let us first consider the low-temperature case, where adatoms cannot overcome the asymmetric barrier at the island corners sufficiently frequently and thus the islands cannot reach their equilibrium shape. ${ }^{18}$ In this case, an island decays along the $x$ and $y$ directions independently. For extracting the scaling laws we consider two regimes.

(i) In the diffusion-limited regime, $\left(D_{x} D_{y}\right)^{-1 / 2} £$ in the first term of the denominator in Eq. (5) is much larger than the other two terms. Thus the time dependence of the island lengths $L_{x}$ and $L_{y}$ is given by

$$
\begin{gathered}
\frac{d L_{x}}{d t}=-\frac{\pi \rho_{x, \infty}^{e q} \gamma_{x} \Omega^{2} C_{0}}{k T} \frac{1}{L_{y} L_{x}} \\
\frac{d L_{y}}{d t}=-\frac{2 \rho_{x, \infty}^{e q} \gamma_{x} \Omega^{2} C_{0}}{k T}\left(\frac{D_{y}}{D_{x}}\right)^{1 / 4} \frac{1}{L_{y}^{1 / 2} L_{x}^{3 / 2}}
\end{gathered}
$$

with $\quad C_{0}=\sqrt{D_{x} D_{y}} /\left(\operatorname{arccosh}\left(b_{R} / c\right)-\operatorname{arccosh}\left\{\left[L_{v} /\left(L_{v}\right.\right.\right.\right.$ $\left.\left.\left.\left.-L_{u}\right)\right]^{1 / 2}\right\}\right) . C_{0}$ has only a weak dependence on $L_{x}\left(L_{y}\right)$ and is assumed to be a constant. In the case of a strong diffusion anisotropy $\left(D_{y} \ll D_{x}\right), d L_{y} / d t$ is close to 0 and thus

$$
L_{y}=\text { const }
$$

while $L_{x}$ is given by

$$
L_{x}=\left(\frac{2 \pi \rho_{x, \infty}^{e q} \gamma_{x} \Omega^{2} C_{0}}{k T L_{y}}\right)^{1 / 2}\left(t_{0}-t\right)^{1 / 2} .
$$

(ii) In the attachment-detachment-limited regime, $K_{x}$ and $K_{y}$ are small and thus the third term of the denominator in Eq. (5) is much larger than the first two. This yields

$$
\begin{gathered}
\frac{d L_{x}}{d t}=-\frac{\pi \rho_{x, \infty}^{e q} \gamma_{x} \Omega^{2} K_{x}}{k T} \frac{1}{L_{x}}, \\
\frac{d L_{y}}{d t}=-\frac{2 \rho_{x, \infty}^{e q} \gamma_{x} \Omega^{2} K_{x}}{k T}\left(\frac{D_{y}}{D_{x}}\right)^{1 / 4} \frac{L_{y}^{1 / 2}}{L_{x}^{3 / 2}} .
\end{gathered}
$$

Analogous to the derivation of Eqs. (8) and (9), the scaling laws for the strongly anisotropic case are

$$
\begin{gathered}
L_{y}=\text { const }, \\
L_{x}=\left(\frac{2 \pi \rho_{x, \infty}^{e q} \gamma_{x} \Omega^{2} K_{x}}{k T}\right)^{1 / 2}\left(t_{0}-t\right)^{1 / 2} .
\end{gathered}
$$

Equations (6)-(13) describe the decay of the island. The length $L_{x}$ and width $L_{y}$ of the island follow simple power laws, i.e., $L_{x} \propto\left(t_{0}-t\right)^{1 / 2}$ and $L_{y} \propto\left(t_{0}-t\right)^{0}$. Thus the island decays quasi-one-dimensionally, i.e., the island length $L_{x}$ decreases continuously in the fast-decay direction, while the island width $L_{y}$ keeps approximately constant in the slow-

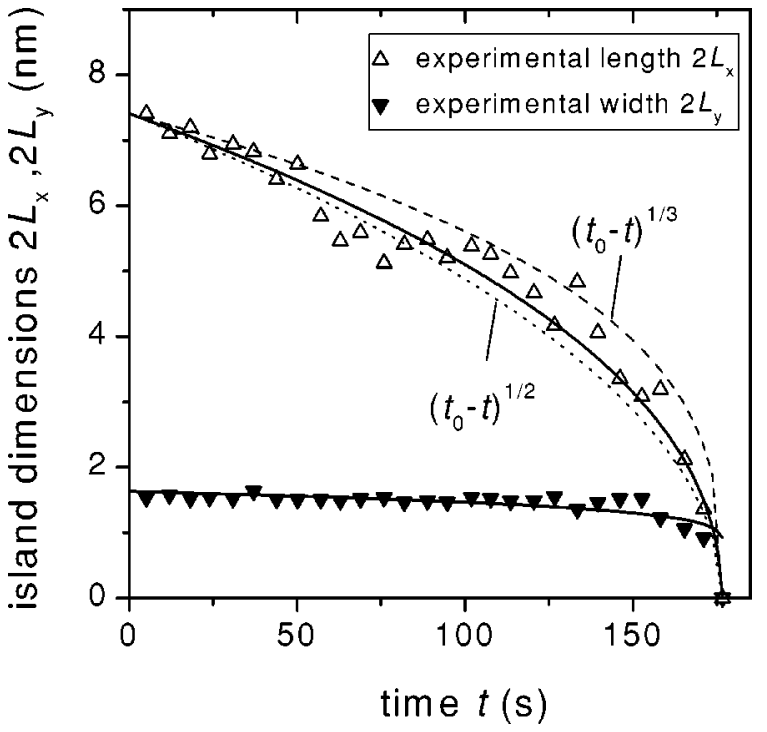

FIG. 2. Comparison of the time evolution of the island dimensions measured experimentally (symbols; taken from Ref. 11) with our analytical model. The solid line is predicted by Eqs. (6) and (7) using the diffusion anisotropy $D_{x} / D_{y}=410$ (see text for details). The dotted and dashed lines show the limiting cases $\propto\left(t_{0}-t\right)^{1 / 2}$ and $\propto\left(t_{0}-t\right)^{1 / 3}$, respectively.

decay direction until the island almost disappears. Furthermore, the exponent $\beta$ in the fast-decay direction is always $1 / 2$, independent of the rate limiting process. This conclusion is in contrast to the case for isotropic systems. Thus for anisotropic systems at low temperatures the value of $\beta$ does not allow conclusion if the decay is diffusion or attachmentdetachment limited. Equations (6) and (10) provide nevertheless a method to discriminate between the different regimes by measuring the decay of islands with different widths. Only in the diffusion-limited regime does the decay rate depend on the island width $L_{y}$. This cannot be predicted within the framework of the conventional Ostwald ripening continuum theory in a $1 \mathrm{D}$ system. ${ }^{19}$

Next we consider the high-temperature case, where atoms can cross the corners of the islands along their perimeters rather easily, ${ }^{18}$ and the islands evolve into their equilibrium shape defined by the aspect ratio $L_{x} / L_{y}=\gamma_{x} / \gamma_{y}=\lambda$. This condition is fulfilled, e.g., above $220 \mathrm{~K}$ on $\mathrm{Ag}(110)$ surfaces, where atoms are able to diffuse along the $\langle 110\rangle$ and $\langle 100\rangle$ oriented steps as well as across the corners. ${ }^{11}$ Therefore, the islands decay two dimensionally by keeping the aspect ratio constant. Thus $\alpha$ in Eq. (5) is also constant. Adding Eqs. (3) and (4) yields the decay rate $d L_{x} / d t=-(\pi \lambda \Re) /\left(4 L_{x}\right)$ from which one obtains $L_{x} \propto\left(t_{0}-t\right)^{\beta}$, with $\beta$ equal to $1 / 2$ and $1 / 3$ for the attachment-detachment-limited and for diffusionlimited cases, respectively. This result is similar to the isotropic case.

We now apply our theory to the recent experiments on the decay of $\mathrm{Ag}$ islands on $\operatorname{Ag}(110)$ surfaces, where a transition from a $1 \mathrm{D}$ to a $2 \mathrm{D}$ decay mode at about $220 \mathrm{~K}$ has been observed. ${ }^{11}$ Our result is in qualitative agreement with the existence of such a transition. However, Morgenstern et al. ${ }^{11}$ 
used a scaling law with $\beta$ equal to $1 / 3$ in analyzing their data even at low temperatures (within the 1D decay mode). This approach disagrees with our present theory yielding a $\beta$ value of $1 / 2$ independent of the decay regime. Based on our current theory, we can reevaluate the experimental data of Ref. 11 in Fig. 2, which shows the time evolution of the width and length of one particular island. The experimental data is shown as symbols. First, we fitted simultaneously the experimental time dependence of the island width and length with the more accurate Eqs. (6) and (7) using a diffusion anisotropy $D_{x} / D_{y}$ of about 410 at $199 \mathrm{~K}$ (based on the diffusion barriers for in-channel and cross-channel migration of $0.279 \mathrm{eV}$ and $0.394 \mathrm{eV}$, respectively ${ }^{11}$ ) and using the assumption of equal vibrational attempt frequencies in both directions. This fit yields the solid lines in Fig. 2. Note the only fit parameter is the prefactor $\left(\pi \rho_{x, \infty}^{e q} \gamma_{x} \Omega^{2} C_{0}\right) /(k T)$, which was obtained to be $0.028 \mathrm{~nm}^{3} \mathrm{~s}^{-1}$. Furthermore, we included a dotted and a dashed line representing the $\left(t_{0}\right.$ $-t)^{1 / 2}$ and $\left(t_{0}-t\right)^{1 / 3}$ scaling laws, respectively (using the same prefactor and $t_{0}$ time). Obviously, the experimental data can be described very well with the present theory and the experimental data points are closer to $\beta$ being equal to $1 / 2$ than to $1 / 3$.

In fact Eqs. (10) and (11) can also fit the data equally well, since they have the same functional form as Eqs. (6) and (7). The only difference lies in the fact that in the diffusion-limited regime the island length is a function of the island width [Eq. (9)], which is not the case for the attachment-detachment-limited regime [Eq. (13)]. Thus, if only isolated island data are investigated, both regimes de- scribe the data equally well, but once the decay is measured as a function of the island width, a clear distinction between the two regimes is possible. Finally, reversing the analysis, it is also possible to extract the diffusion anisotropy from the experiment using the diffusion anisotropy as a fit parameter for larger data sets.

In conclusion, we have developed an analytical description of the decay of 2D islands on surfaces with a pronounced diffusion anisotropy. We derived the generic scaling law $L \propto\left(t_{0}-t\right)^{\beta}$ describing the island dimensions versus time. For the low-temperature regime, where atoms crossing around island corners is infrequent, the island decays quasione-dimensionally, i.e., $\beta$ is 0 and $1 / 2$ for the slow- and fast-decay directions, respectively. This is valid for both the diffusion- and the attachment-detachment-limited regimes, in contrast to the case of island decay on isotropic surfaces. In the high-temperature regime with frequent corner crossings, we obtained analogous scaling laws to those for the isotropic case, namely, $\beta$ allows an extraction of the physical process limiting the decay rate. Applications of our predictions to recent experimental data for island decay of $\mathrm{Ag} / \mathrm{Ag}(110)$ have resulted in an improved agreement between theory and experiment.

We thank Ying Zhang for helpful discussions. This work has been supported in part by the Natural Science Foundation of China, the National Key Project of Basic Research Grant No. (G2000067103), and ORNL, managed by UTBattell, LLC, for the U.S. DOE under Contract No. DEAC05-00OR22725.
${ }^{1}$ A. Ichimiya et al., Phys. Rev. Lett. 84, 3662 (2000).

${ }^{2}$ M. Li et al., Phys. Rev. Lett. 86, 2345 (2001).

${ }^{3}$ K. Morgenstern, G. Rosenfeld, and G. Comsa, Phys. Rev. Lett. 76, 2113 (1996).

${ }^{4}$ J.-M. Wen et al., Phys. Rev. Lett. 76, 652 (1996).

${ }^{5}$ J.B. Hannon et al., Phys. Rev. Lett. 79, 2506 (1997).

${ }^{6}$ W.-W. Pai et al., Phys. Rev. Lett. 79, 3210 (1997).

${ }^{7}$ C. Klünker et al., Phys. Rev. B 58, R7556 (1998).

${ }^{8}$ G.S. Icking-Konert, M. Giesen, and H. Ibach, Surf. Sci. 398, 37 (1998).

${ }^{9}$ M. Giesen, G.S. Icking-Konert, and H. Ibach, Phys. Rev. Lett. 80, 552 (1998).

${ }^{10}$ K. Morgenstern et al., Phys. Rev. Lett. 80, 556 (1998).

${ }^{11}$ K. Morgenstern et al., Phys. Rev. Lett. 83, 1613 (1999).

${ }^{12}$ J.G. McLean et al., Phys. Rev. B 55, 1811 (1997), and references therein.

${ }^{13}$ S. Rusponi et al., Surf. Sci. 440, 451 (1999).

${ }^{14}$ M.J. Rost, R. van Gastel, and J.W.M. Frenken, Phys. Rev. Lett. 84, 1966 (2000).

${ }^{15}$ K. Morgenstern, E. Laegsgaard, and F. Besenbacher, Phys. Rev. Lett. 86, 5739 (2001).

${ }^{16}$ F. Hontinfinde, R. Ferrando, and A.C. Levi, Surf. Sci. 366, 306 (1996).

${ }^{17}$ A. Pimpinelli and J. Villain, Physics of Crystal Growth (Cambridge University Press, Cambridge, England, 1998), p. 43.

${ }^{18}$ J. Zhong et al., Phys. Rev. B 63, 113403 (2001).

${ }^{19}$ L. D. Laudau and E. M. Lifshitz, Course of Theoretical Physics, Statistical Physics (Pergamon Press, New York, 1959), Vol. 5, p. 482. 